8. Rohde JA, Noar SM, Mendel JR, Hall MG, Baig SA, Ribisl KM, et al. E-cigarette health harm awareness and discouragement: implications for health communication. Nicotine Tob Res [online ahead of print] 9 Oct 2019; DOI:10.1093/ntr/ntz194.

Copyright (C) 2020 by the American Thoracic Society

Check for updates

\section{Alveolar Macrophage Immunometabolism and Lung Function Impairment in Smoking and Chronic Obstructive Pulmonary Disease}

\section{To the Editor:}

Metabolic plasticity involving shifts between mitochondrial respiration and glycolysis is emerging as a crucial component of innate immune cell function. The number of alveolar macrophages (AMs), the most abundant antigen-presenting cells in the lung, is increased in the lungs of individuals with chronic obstructive pulmonary disease (COPD), associating with disease severity and areas of lung destruction $(1,2)$. However, COPD AMs exhibit dysfunctional responses to infection and have lower phagocytic and bactericidal activity (3-5). Metabolism is a key determinant of immune cell function and is a vital component governing macrophage adaptation and responses. Metabolic reprogramming involving a shift from ATP production via the mitochondrial electron transport chain, toward anaerobic respiration via glycolysis, is an important proinflammatory effector function of macrophages (6). The presence and nature of any functional shifts in AM metabolic activity in COPD has not been determined.

In this study, healthy nonsmokers, smokers, and individuals with COPD, all $\geqslant 8$ weeks free of an acute exacerbation, underwent bronchoscopy with BAL and collection of AMs, as previously described (4). In BAL adherence-purified AMs, we first assessed the mitochondrial-related transcriptomic profile of AMs from 22 individuals with COPD, 42 smokers and 24 healthy control individuals, by HG-U133 Plus 2.0 microarray (Affymetrix; deposited as GSE130928, some of whom participated in a prior study [4]), normalized using the Robust Multi-Array method and filtered on the 1,938 mitochondrial-related probesets (Mitocarta2.0). Agecorrected analysis of covariance with Benjamini-Hochberg-corrected $P<0.05$ considered significant, followed by grouping based on gene ontology biological processes, revealed significant alterations in mitochondria-related gene expression in response to cigarette smoke exposure and in COPD. A total of 105 genes (9\%) were significantly differentially expressed in smokers versus nonsmoker control

Supported by NIH grants R00-20 HL125899 (S.M.C.), HL113443 (R.G.C.), HL1189541 (R.G.C.), and HL094284 (M.R.R.).

Author Contributions: S.L.O'B. and S.M.C. conceived and designed the study; S.L.O'B. and R.J.K. obtained samples and clinical data; S.A.K. and S.M.C. performed the experiments; C.O., J.S., M.R.R., and K.V.B. performed the data analyses; R.G.C. provided critical discussions for data interpretation; S.L.O'B. and S.M.C. wrote the manuscript; and all coauthors reviewed and approved the final manuscript.

Originally Published in Press as DOI: 10.1164/rccm.201908-1683LE on November 21, 2019 individuals with an enrichment of genes related to glutathione metabolism, mitochondrial transport, protein localization and translation, pyruvate metabolism, the tricarboxylic acid cycle, the electron transport chain, and fatty acid oxidation (false discovery rate-adjusted $P<0.05)$. A total of 129 genes $(11 \%)$ were significantly altered in smokers with COPD versus nonsmoker control individuals, whereas 149 genes (13\%) were significantly differentially expressed in smokers with COPD versus healthy smokers and were enriched for processes related to intrinsic apoptotic signaling pathways, mitochondrial fission, calcium transport, mitochondrial biogenesis, the NLRP1 inflammasome, pyruvate metabolism, complex I biogenesis, and fatty acid oxidation (false discovery rateadjusted $P<0.05$; Figure 1A).

To assess function, we prospectively assessed mitochondrial and glycolytic metabolic activity in the AMs of 12 individuals with COPD, 18 smokers, and 23 healthy control individuals, using a Seahorse Bioanalyzer. Basal oxygen consumption rates and extracellular acidification rates (ECAR) of AMs were similar among nonsmoker control individuals, smokers, and patients with COPD; however, the coupling efficiency in AMs from smokers and individuals with COPD was significantly lower when compared with that of control individuals. Although ATP production was similar between phenotypes, proton leak was significantly higher in AMs from smokers and individuals with COPD versus nonsmoker control individuals. Maximal respiration and the spare respiratory capacity (reserve capacity) were significantly lower in AMs from patients with COPD than in those from smokers. The Bioenergetic Health Index (representing the composite mitochondrial profile) of AMs from both smokers and individuals with COPD was also significantly lower than that of healthy control individuals (Figure $1 \mathrm{~B}$ and Table 1).

If the spare respiratory capacity is decreased and the threshold activity cannot be met, glycolysis may be stimulated to meet cellular energetic needs. In this study, the rate of glycolysis-specific proton efflux (GlycoPER) was significantly higher in AMs from smokers versus those from healthy control individuals, and smokers had significantly higher post-2G-GlycoPER (the nonglycolytic, nonmitochondrial cellular ECAR) versus healthy control individuals. In contrast, patients with COPD had significantly lower compensatory glycolysis, post-2DG-GlycoPER, and mitochondrial-specific glycolysis versus smokers (Figure 1C and Table 1).

Finally, we examined whether the above AM characteristics associated with clinical parameters including $\mathrm{FEV}_{1} \%$ predicted and $\mathrm{DL}_{\mathrm{CO}}$ by implementing a linear model that also included age, sex, and subject number. Maximal respiration, spare respiratory capacity, Bioenergetic Health Index, compensatory glycolysis, and post-2DG correlated with spirometric impairment, as defined by $\mathrm{FEV}_{1} \%$ predicted (Figures $1 \mathrm{D}$ and $1 \mathrm{E}$ ). In addition, the expression of 219 (19\%) mitochondrial-related genes significantly correlated with $\mathrm{FEV}_{1} \%$ predicted in control individuals, healthy smokers, and COPD smokers (Figure $1 \mathrm{~F}$ ). A low $\mathrm{DL}_{\mathrm{CO}}$ in individuals with normal $\mathrm{FEV}_{1} \%$ predicted has been linked to the development of COPD (7). Here, AM proton leak was numerically higher (mean, 7.38 vs. $4.44 \mathrm{pmol} / \mathrm{min} / \mathrm{ng} \mathrm{DNA} P=0.21$ ), and coupling efficiency was lower $(0.82$ vs. $0.87 \mathrm{pmol} / \mathrm{min} / \mathrm{ng} \mathrm{DNA} ; P=0.21)$, in smokers with a low $\mathrm{DL}_{\mathrm{CO}}$ than in healthy smokers. Smokers with a low $\mathrm{DL}_{\mathrm{CO}}$ also had significantly lower Bioenergetic Health Index when 
A

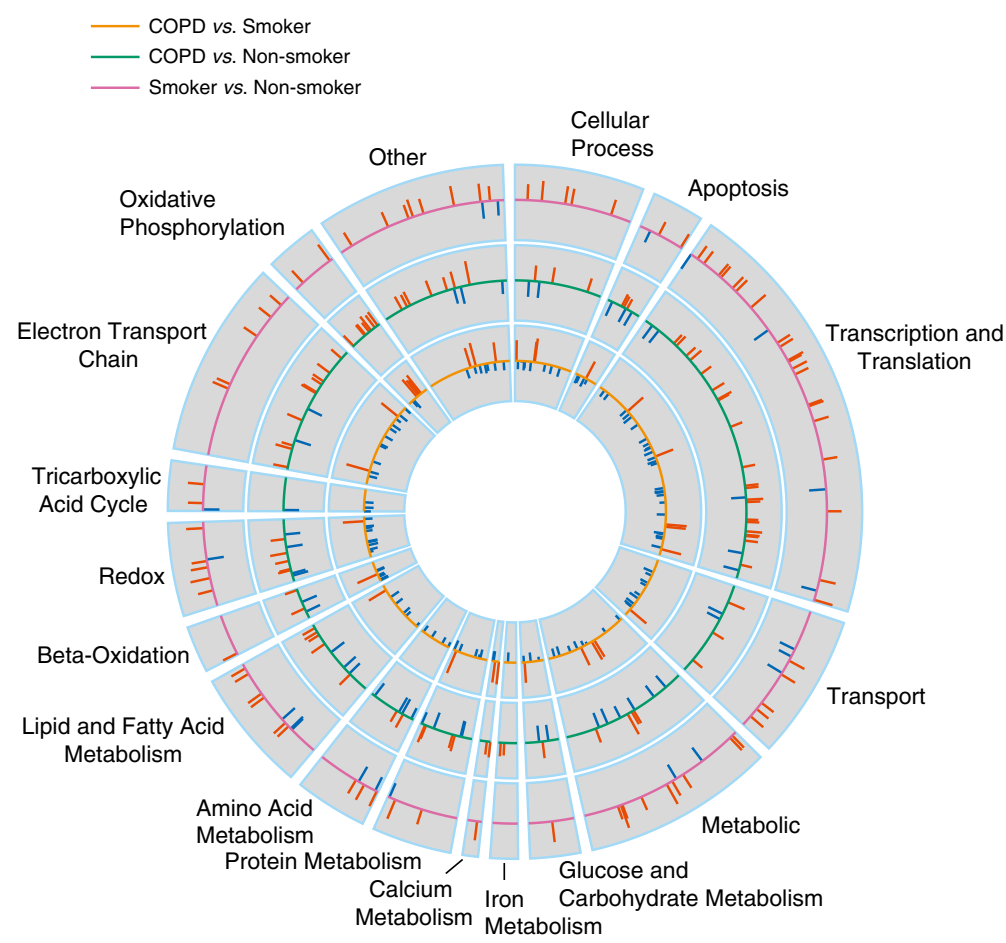

B

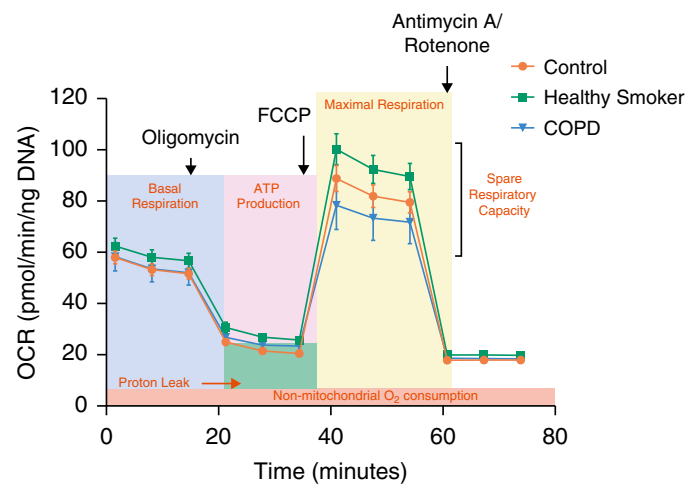

C

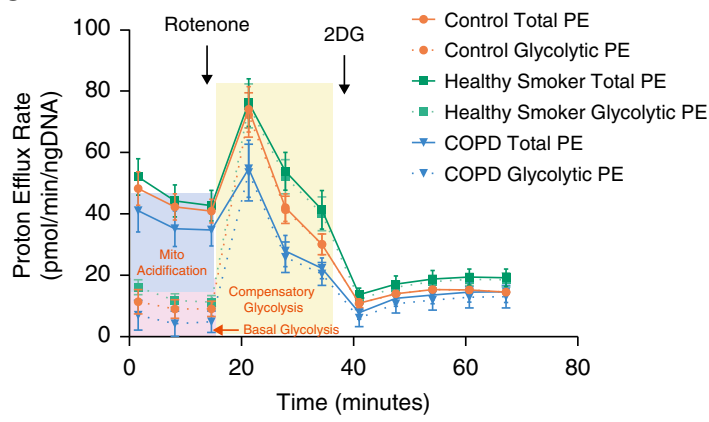

D
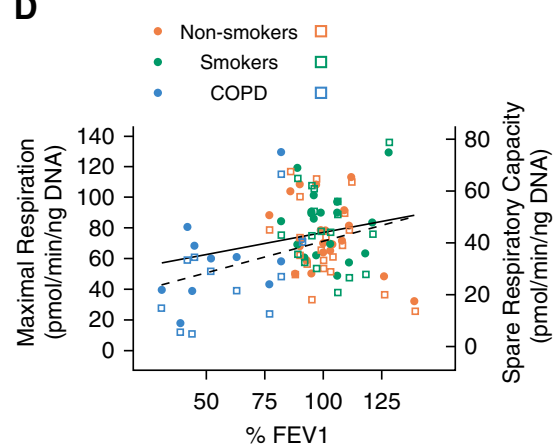

Maximal Respiration $\hat{\beta}=0.27, p=0.054$
Adj age and sex, $\hat{\beta}=0.31, p=0.057$
- - Spare Respiratory $\hat{\beta}=0.29, p=0.018$
Adj age and sex, $\hat{\beta}=0.25, p=0.02$
E

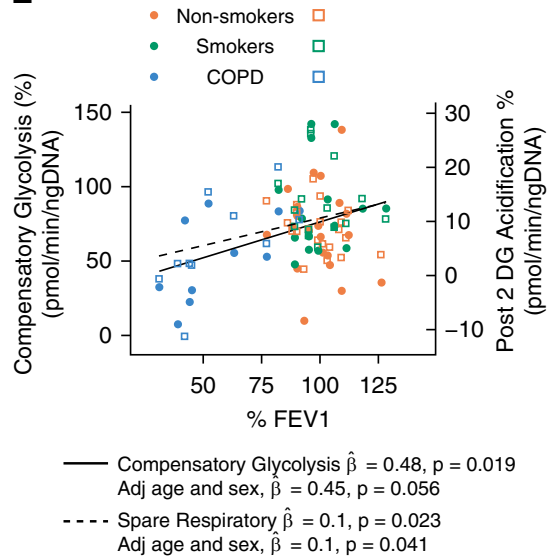

$\mathbf{F}$

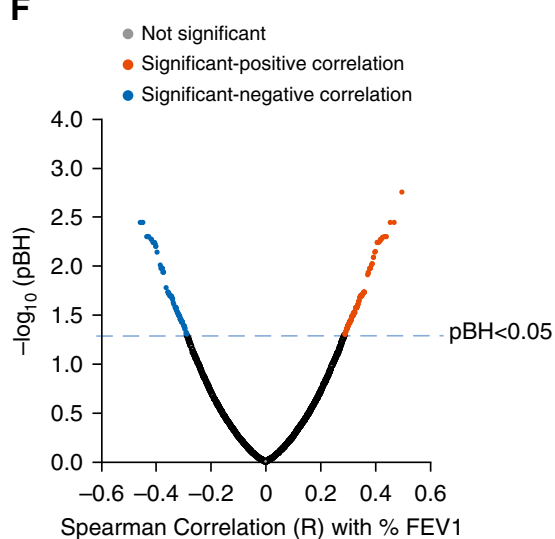

Figure 1. Mitochondrial gene expression and metabolic activity associate with $\mathrm{FEV}_{1} \%$ predicted decline in smokers and individuals with chronic obstructive pulmonary disease (COPD). (A) Circos plot of changes in alveolar macrophage (AM) mitochondria-related gene expression (analysis of covariance with Benjamini-Hochberg-corrected $P<0.05$ considered significant) grouped by gene ontology biological process; the height of the bars corresponds to the $\log _{2}$ fold change (FC) (red bars = positive FC, blue bars = negative FC) between phenotype groups with smokers $(n=42)$ versus nonsmokers $(n=24)$ in pink (outside circle), smokers with COPD $(n=22)$ versus nonsmokers in green (middle circle), and smokers with COPD versus smokers in orange (inner circle). (B) Oxygen consumption rates normalized to DNA in AMs from nonsmoker controls $(n=23)$, smokers $(n=18)$, and subjects with COPD $(n=12)$ when treated sequentially with oligomycin $(1 \mu \mathrm{M})$, carbonyl cyanide 4-(trifluoromethoxy) phenylhydrazone (1.5 $\mu \mathrm{M})$, and rotenone/antimycin $\mathrm{A}(0.5 \mu \mathrm{M})$. (C) Total proton efflux rates and the proton efflux rates attributed to glycolysis normalized to DNA in AMs from nonsmoker control individuals $(n=20)$, smokers $(n=16)$, and subjects with COPD $(n=11)$ when treated sequentially with rotenone/antimycin A $(0.5 \mu \mathrm{M})$ and 2 -deoxyD-glucose (2DG; $50 \mu \mathrm{M})$. Data ( $B$ and $C$ ) are presented as the mean \pm SEM for control individuals, healthy smokers, and patients with COPD, based on six technical replicates for each subject. $(D$ and $E$ ) Seahorse parameters including maximal respiration $(D)$, spare respiratory capacity $(D)$, compensatory glycolysis $(E)$, and post-2DG acidification $(E)$ of AMs from nonsmoker control individuals $(n=20-21)$, smokers $(n=16-18)$, and subjects with COPD $(n=11-12)$ and association with $\mathrm{FEV}_{1} \%$ predicted. In panels $D$ and $E$, we present the estimated slope $(\hat{\beta})$ from both a simple linear model as well as one adjusted for age and sex to test for association. $(F)$ Volcano plot of mitochondrial-related genes significantly associated with $\mathrm{FEV} \mathrm{V}_{1} \%$ predicted in $\mathrm{AMs}$ of nonsmokers $(n=24)$, healthy smokers $(n=42)$, and individuals with COPD $(n=22)$. The $y$-axis depicts the $-\log 10$ of Benjamini-Hochberg-corrected $P$ value; the $x$-axis depicts the Spearman Correlation $(R)$ between gene expression and $\mathrm{FEV}_{1} \%$ predicted; red, significantly upregulated; blue, significantly downregulated; and gray, not significant. Adj=adjusted; FCCP = carbonyl cyanide 4-(trifluoromethoxy) phenylhydrazone; OCR=oxygen consumption rate; $\mathrm{PE}=$ proton efflux. 
Table 1. Demographics and Seahorse Parameters of Study Subjects

\begin{tabular}{|c|c|c|c|c|c|c|}
\hline Parameter & Nonsmokers & Smokers* & COPD $^{\dagger}$ & $\begin{array}{c}\text { Smokers vs. } \\
\text { Nonsmokers } \\
P \text { Value }\end{array}$ & $\begin{array}{l}\text { COPD vs. } \\
\text { Nonsmokers } \\
P \text { Value }\end{array}$ & $\begin{array}{c}\text { COPD vs. Smokers } \\
P \text { Value }\end{array}$ \\
\hline Transcriptome cohort, $n$ & 24 & 42 & 22 & & & \\
\hline Sex, M/F, $n$ & $18 / 6$ & $29 / 13$ & $18 / 4$ & $0.6\left(0.84^{\ddagger}\right)$ & $0.5\left(0.62^{\ddagger}\right)$ & $0.2\left(0.23^{\ddagger}\right)$ \\
\hline Age, yr & $40 \pm 8$ & $43 \pm 8$ & $54 \pm 8$ & $0.51\left(0.52^{\ddagger}\right)$ & $<0.001\left(<0.001^{\S}\right)$ & $<0.001\left(<0.001^{\S}\right)$ \\
\hline Race, $\mathrm{B} / \mathrm{W} / \mathrm{H}, n$ & $15 / 6 / 3$ & $23 / 13 / 6$ & $5 / 13 / 4$ & $0.8\left(0.84^{f^{\prime}}\right)$ & $0.05\left(0.97^{\ddagger}\right)$ & $0.05\left(0.72^{\ddagger}\right)$ \\
\hline Smoking, pack-years & - & $27 \pm 17$ & $43 \pm 28$ & - & - & $0.002\left(<0.001^{\S}\right)$ \\
\hline $\mathrm{FEV}_{1}$ & $102 \pm 12$ & $105 \pm 13$ & $85 \pm 23$ & $0.79\left(0.73^{\S}\right)$ & $0.001\left(<0.001^{\S}\right)$ & $<0.001\left(<0.001^{\S}\right)$ \\
\hline $\mathrm{D} \mathrm{L}_{\mathrm{CO}}$ & $81 \pm 6$ & $80 \pm 6$ & $62 \pm 8$ & $0.99\left(0.92^{\S}\right)$ & $<0.001\left(<0.001^{\S}\right)$ & $<0.001\left(<0.001^{\S}\right)$ \\
\hline GOLD stage, I/II/III/IV\| & - & - & $13 / 7 / 2 / 0$ & - & - & - \\
\hline $\begin{array}{l}\text { Medications: } \\
\text { ICS/LAMA/Statin, } n\end{array}$ & $0 / 0 / 0$ & $0 / 0 / 0$ & $1 / 1 / 2$ & - & - & - \\
\hline Seahorse cohort, $n$ & 23 & 18 & 12 & & & \\
\hline Sex, M/F, $n$ & $10 / 13$ & $12 / 6$ & $9 / 3$ & $0.1\left(0.38^{\ddagger}\right)$ & $0.07\left(0.26^{\ddagger}\right)$ & $0.9\left(0.9^{\ddagger}\right)$ \\
\hline Age, yr & $38 \pm 11$ & $48 \pm 11$ & $62 \pm 7$ & $0.010\left(0.001^{\S}\right)$ & $<0.001\left(<0.001^{\S \S}\right)$ & $0.002\left(0.001^{\S}\right)$ \\
\hline Race, B/W/H/O, $n$ & $11 / 4 / 3 / 5$ & $11 / 2 / 5 / 0$ & $3 / 7 / 1 / 1$ & $0.3\left(0.16^{\ddagger}\right)$ & $0.2\left(0.6^{\ddagger}\right)$ & $0.1\left(0.026^{\ddagger}\right)$ \\
\hline Smoking, pack-years & - & $22 \pm 15$ & $36 \pm 23$ & - & - & 0.05 \\
\hline $\mathrm{FEV}_{1}$ & $101 \pm 13$ & $102 \pm 12$ & $58 \pm 20$ & $0.99\left(0.96^{\S}\right)$ & $<0.001\left(<0.001^{\S}\right)$ & $<0.001\left(<0.001^{\S}\right)$ \\
\hline DLCO & $94 \pm 12$ & $80 \pm 15$ & $71 \pm 22$ & $0.022\left(0.027^{\S}\right)$ & $<0.001\left(0.008^{\S}\right)$ & $0.28\left(0.36^{\S}\right)$ \\
\hline GOLD stage, I/II/III/IV\| & - & - & $4 / 2 / 6 / 0$ & - & - & - \\
\hline $\begin{array}{l}\text { Medications: } \\
\text { ICS/LAMA/Statin, } n\end{array}$ & 0/0/0 & $0 / 0 / 0$ & $7 / 5 / 1$ & - & - & - \\
\hline \multicolumn{7}{|l|}{ Seahorse parameters } \\
\hline $\begin{array}{l}\text { Basal respiration, } \\
\text { pmol/min/ng DNA }\end{array}$ & $35 \pm 8$ & $37 \pm 8$ & $35 \pm 13$ & $0.75\left(0.81^{\S}\right)$ & $0.97\left(0.95^{\S}\right)$ & $0.68\left(0.67^{\S}\right)$ \\
\hline Coupling efficiency, \% & $0.9 \pm 0.04$ & $0.8 \pm 0.07$ & $0.8 \pm 0.05$ & $0.001\left(0.001^{\S}\right)$ & $0.004\left(0.011^{\S}\right)$ & $0.98\left(0.91^{\S}\right)$ \\
\hline $\begin{array}{l}\text { ATP production, } \\
\mathrm{pmol} / \mathrm{min} / \mathrm{ng} \text { DNA }\end{array}$ & $32 \pm 7$ & $31 \pm 6$ & $29 \pm 9$ & $0.95\left(0.91^{\S}\right)$ & $0.48\left(0.49^{\S}\right)$ & $0.66\left(0.58^{\S}\right)$ \\
\hline $\begin{array}{l}\text { Proton leak, } \\
\text { pmol/min/ng DNA }\end{array}$ & $3 \pm 2$ & $6 \pm 3$ & $6 \pm 4$ & $0.009\left(0.008^{\S}\right)$ & $0.08\left(0.13^{\S}\right)$ & $0.91\left(0.98^{\S}\right)$ \\
\hline $\begin{array}{l}\text { Max respiration, } \\
\mathrm{pmol} / \mathrm{min} / \mathrm{ng} \text { DNA }\end{array}$ & $75 \pm 22$ & $83 \pm 21$ & $61 \pm 29$ & $0.53\left(0.61^{\S}\right)$ & $0.25\left(0.36^{\S}\right)$ & $0.045\left(0.046^{\S}\right)$ \\
\hline $\begin{array}{l}\text { Reserve capacity, } \\
\mathrm{pmol} / \mathrm{min} / \mathrm{ng} \text { DNA }\end{array}$ & $40 \pm 15$ & $45 \pm 15$ & $26 \pm 18$ & $0.48\left(0.56^{\S}\right)$ & $0.08\left(0.16^{\S}\right)$ & $0.008\left(0.008^{\S}\right)$ \\
\hline $\begin{array}{l}\text { Non-mito OCR, } \\
\mathrm{pmol} / \mathrm{min} / \mathrm{ng} \text { DNA }\end{array}$ & $17 \pm 4$ & $20 \pm 5$ & $18 \pm 5$ & $0.16\left(0.48^{\S}\right)$ & $0.96\left(0.74^{\S}\right)$ & $0.40\left(0.16^{\S}\right)$ \\
\hline $\begin{array}{l}\text { Bioenergetic Health } \\
\text { Index, AU }\end{array}$ & $1.3 \pm 0.2$ & $1.1 \pm 0.2$ & $0.9 \pm 0.3$ & $0.02\left(0.09^{\S}\right)$ & $<0.001\left(0.004^{\S}\right)$ & $0.027\left(0.11^{\S}\right)$ \\
\hline PER, \% & $39 \pm 12$ & $49 \pm 23$ & $35 \pm 17$ & $0.25\left(0.10^{\S}\right)$ & $0.79\left(0.85^{\S}\right)$ & $0.14\left(0.56^{\S}\right)$ \\
\hline $\begin{array}{l}\text { Mito PER, pmol/min/ng } \\
\text { DNA }\end{array}$ & $9 \pm 58$ & $5 \pm 17$ & $-0.2 \pm 28$ & $0.95\left(0.99^{\S}\right)$ & $0.84\left(0.99^{\S}\right)$ & $0.96\left(0.99^{\S}\right)$ \\
\hline $\begin{array}{l}\text { Compensatory } \\
\text { glycolysis, \% }\end{array}$ & $71 \pm 29$ & $86 \pm 31$ & $53 \pm 29$ & $0.32\left(0.26^{\S}\right)$ & $0.30\left(0.81^{\S}\right)$ & $0.031\left(0.12^{\S}\right)$ \\
\hline $\begin{array}{l}\text { Basal glycolysis, } \\
\mathrm{pmol} / \mathrm{min} / \mathrm{ng} \text { DNA }\end{array}$ & $8 \pm 9$ & $14 \pm 12$ & $5 \pm 11$ & $0.26\left(0.20^{\S}\right)$ & $0.76\left(0.99^{\S}\right)$ & $0.13\left(0.36^{\S}\right)$ \\
\hline Post-2DG GlycoPER, \% & $9 \pm 4$ & $14 \pm 7$ & $6 \pm 9$ & $0.06\left(0.050^{\S}\right)$ & $0.45\left(0.86^{\S}\right)$ & $0.010\left(0.036^{\S}\right)$ \\
\hline
\end{tabular}

Definition of abbreviations: $2 \mathrm{DG}=$ deoxyglucose; $\mathrm{AU}=$ arbitrary units; $\mathrm{B}=$ black; $\mathrm{COPD}=$ chronic obstructive pulmonary disease; $\mathrm{GOLD}=\mathrm{Global}$ Initiative for Chronic Obstructive Lung Disease; $\mathrm{H}=$ Hispanic; ICS = inhaled corticosteroid; LAMA = long-acting muscarinic antagonists; mito=mitochondrial; $\mathrm{O}=$ other; $\mathrm{OCR}=$ oxygen consumption rate; $\mathrm{PER}=$ proton efflux rate; $\mathrm{W}=$ white.

Demographic data are presented as mean \pm SD unless otherwise specified.

${ }^{*}$ All smokers in the transcriptome cohort are healthy smokers with normal lung function; the seahorse cohort includes $n=7$ healthy smokers with norma lung function and $n=11$ smokers with normal spirometry and low $\mathrm{DL}_{\mathrm{co}}$.

${ }^{\dagger}$ All patients with COPD in the transcriptome cohort are current smokers; the seahorse cohort includes $n=6$ current smokers and $n=6$ ex-smokers with COPD.

${ }^{\ddagger}$ Adjustment for age and sex implemented with Cochran-Mantel-Haenszel chi-square test, dichotomizing age $<50$ years.

${ }^{\S}$ Adjustment for age and sex implemented with a linear model using the Tukey correction.

"Based on GOLD criteria.

compared with healthy control individuals (1.04 vs. $1.34 \mathrm{AU}$; $P=0.013)$.

In summary, we identified changes in the mitochondrial gene signature and immunometabolic behavior of AMs from smokers and individuals with COPD, with lower mitochondrial respiration and defective compensatory glycolysis observed in patients with COPD when compared with smokers, correlating with a lower $\mathrm{FEV}_{1} \%$ predicted. Although understanding the precise biological implications of these associations will require further mechanistic studies, these findings support a hypothesis 
that dysfunctional AM immunometabolic activities may impair normal responses to respiratory pathogens, which may contribute to chronic airway persistence of bacteria and lung function decline in COPD. These findings may also provide insight into the pathobiology underlying the increased rate of progression observed in smokers with a low $\mathrm{DL}_{\mathrm{CO}}$ to the $\mathrm{FEV}_{1} \%$ predicted impairment present in COPD.

Our findings are consistent with prior studies demonstrating that BAL AMs in COPD have aberrant macrophage responses associated with dysfunctional mitochondria and that smoker BAL macrophages have lower respiratory capacities (8-10). Here, we find that AMs from smokers have a higher basal glycolytic rate and more nonglycolytic nonmitochondrial ECAR; however, smokers with COPD have greatly reduced compensatory glycolysis and nonglycolytic, nonmitochondrial ECAR, suggesting that AMs in COPD have lost the ability to dynamically compensate for mitochondrial dysfunction by increasing glycolysis. These observations may be explained by lower total AM mitochondrial numbers and mass. However, assessment of AM expression of mitochondrial-encoded genes revealed comparable mitochondrial DNA expression across all groups, suggesting that other factors such as second messenger signaling, cell death, or the differential availability of metabolites/nutrients in the microenvironment of the COPD lung may also play a role.

Limitations of the current study include the relatively small subject number and demographic differences between groups, whereby patients with COPD were significantly older. However, the statistically significant genes identified by our transcriptomic analyses and the significant differences identified between Seahorse parameters and disease stage, as well as the significant differences between Seahorse parameters and $\mathrm{FEV}_{1} \%$ predicted, were adjusted for age (Table 1), thus removing age as a confounding factor in the interpretation. Furthermore, although the protocol used for adherence purification of BAL macrophages results in $88-98 \%$ purity in the phenotypes studied (11-18), postadherence AM purity was not independently confirmed in this cohort. In addition, AMs isolated by adherence from BAL include both resident and monocyte-derived macrophages, and it was not possible to assess whether immunometabolic changes were driven by one or the other of these AM subtypes, whether AM metabolic activity was influenced by ex vivo culture conditions, or whether the uncoupler, carbonyl cyanide 4(trifluoromethoxy) phenylhydrazone, used in the Seahorse assays, induced cell death and influenced the total cell number at the end of the assay.

To conclude, these findings imply that AM

immunometabolism is an important pathobiological phenomenon and suggests that modification of AM cellular metabolism may be a novel therapeutic approach to enhance the innate immune response of the AM in COPD.

\section{Author disclosures are available with the text of this letter at www.atsjournals.org.}

Acknowledgment: The authors thank Dr. Augustine M. K. Choi for critical discussion and insight, Nahla Mohamed for formatting help, and Ashleih Burke, Meghanne Lomibao, and Kihwan Kim for technical support.
Sarah L. O’Beirne, M.D., Ph.D.

Sarah Ann Kikkers, B.A.

Clara Oromendia, M.S.

Jacqueline Salit, Ph.D.

Mahboubeh R. Rostmai, Ph.D.

Karla V. Ballman, Ph.D.*

Robert J. Kaner, M.D.

Ronald G. Crystal, M.D.

Suzanne M. Cloonan, Ph.D. ${ }^{\ddagger}$

Weill Cornell Medicine

New York, New York

ORCID IDs: 0000-0003-2876-0258 (S.L.O'B.); 0000-0001-5301-9926 (S.M.C.).

*K.V.B. is Biostatistician of AJRCCM. Her participation complies with American Thoracic Society requirements for recusal from review and decisions for authored works.

${ }^{\ddagger}$ Corresponding author (e-mail: szc2009@med.cornell.edu).

\section{References}

1. Di Stefano A, Capelli A, Lusuardi M, Balbo P, Vecchio C, Maestrelli $P$, et al. Severity of airflow limitation is associated with severity of airway inflammation in smokers. Am J Respir Crit Care Med 1998;158:1277-1285.

2. Finkelstein R, Fraser RS, Ghezzo H, Cosio MG. Alveolar inflammation and its relation to emphysema in smokers. Am J Respir Crit Care Med 1995;152:1666-1672.

3. Taylor AE, Finney-Hayward TK, Quint JK, Thomas CM, Tudhope SJ, Wedzicha JA, et al. Defective macrophage phagocytosis of bacteria in COPD. Eur Respir J 2010;35:1039-1047.

4. Shaykhiev R, Krause A, Salit J, Strulovici-Barel Y, Harvey BG, O’Connor $\mathrm{TP}$, et al. Smoking-dependent reprogramming of alveolar macrophage polarization: implication for pathogenesis of chronic obstructive pulmonary disease. J. Immunol. 2009;183:2867-2883.

5. Hodge S, Hodge G, Ahern J, Jersmann H, Holmes M, Reynolds PN. Smoking alters alveolar macrophage recognition and phagocytic ability: implications in chronic obstructive pulmonary disease. Am J Respir Cell Mol Biol 2007;37:748-755.

6. Van den Bossche J, O'Neill LA, Menon D. Macrophage immunometabolism: where are we (going)? Trends Immunol 2017;38:395-406.

7. Harvey BG, Strulovici-Barel Y, Kaner RJ, Sanders A, Vincent TL, Mezey $\mathrm{JG}$, et al. Risk of COPD with obstruction in active smokers with normal spirometry and reduced diffusion capacity. Eur Respir J 2015;46: 1589-1597.

8. Gleeson LE, O'Leary SM, Ryan D, McLaughlin AM, Sheedy FJ, Keane J. Cigarette smoking impairs the bioenergetic immune response to mycobacterium tuberculosis infection. Am J Respir Cell Mol Biol 2018; 59:572-579.

9. Belchamber KBR, Singh R, Batista CM, Whyte MK, Dockrell DH, Kilty I, et al.; COPD-MAP consortium. Defective bacterial phagocytosis is associated with dysfunctional mitochondria in COPD macrophages. Eur Respir J 2019;54:1802244.

10. Bewley MA, Preston JA, Mohasin M, Marriott HM, Budd RC, Swales J, et al. Impaired mitochondrial microbicidal responses in chronic obstructive pulmonary disease macrophages. Am J Respir Crit Care Med 2017;196:845-855.

11. Monick MM, Carter AB, Hunninghake GW. Human alveolar macrophages are markedly deficient in REF-1 and AP-1 DNA binding activity. J Biol Chem 1999;274:18075-18080.

12. Flaherty DM, Monick MM, Hinde SL. Human alveolar macrophages are deficient in PTEN: the role of endogenous oxidants. $J$ Biol Chem 2006;281:5058-5064.

13. Heguy A, O'Connor TP, Luettich K, Worgall S, Cieciuch A, Harvey BG, et al. Gene expression profiling of human alveolar macrophages of phenotypically normal smokers and nonsmokers reveals a previously unrecognized subset of genes modulated by cigarette smoking. J Mol Med (Berl) 2006;84:318-328.

14. Kazeros A, Harvey BG, Carolan BJ, Vanni H, Krause A, Crystal RG. Overexpression of apoptotic cell removal receptor MERTK in alveolar macrophages of cigarette smokers. Am J Respir Cell Mol Biol 2008; 39:747-757. 
15. Kaner RJ, Santiago F, Crystal RG. Up-regulation of alveolar macrophage matrix metalloproteinases in HIV1(+) smokers with early emphysema. J Leukoc Biol 2009;86:913-922.

16. Tomlinson GS, Booth H, Petit SJ, Potton E, Towers GJ, Miller RF, et al. Adherent human alveolar macrophages exhibit a transient proinflammatory profile that confounds responses to innate immune stimulation. PLoS One 2012;7:e40348.

17. Poole E, Juss JK, Krishna B, Herre J, Chilvers ER, Sinclair J. Alveolar macrophages isolated directly from human cytomegalovirus (HCMV)-seropositive individuals are sites of HCMV reactivation in vivo. J Infect Dis 2015;211:1936-1942.

18. Nayak DK, Mendez O, Bowen S, Mohanakumar T. Isolation and in vitro culture of murine and human alveolar macrophages. J Vis Exp 2018; (134):57287.

Copyright (C) 2020 by the American Thoracic Society

\section{Check for updates}

\section{Lung Function Decline in Early HIV Infection: Impact of Antiretroviral Drug Timing and Drug Regimen}

\section{To the Editor:}

HIV infection is an independent risk factor for chronic obstructive pulmonary disease (COPD) (odds ratio, 1.14; 95\% confidence interval, 1.05-1.25) (1). Mechanisms remain unclear, but some observational studies have suggested that HIV antiretroviral treatment (ART) might lead to worse lung function and subsequent $\operatorname{COPD}(2,3)$.

We addressed the effects of HIV treatment on lung function in the START (Strategic Timing of Antiretroviral Treatment) Trial Pulmonary Substudy. We previously published initial results from this randomized trial in which 1,026 HIV-positive, ART-naive adults (from 80 sites in 20 high- and low- to middle-income countries) with $\mathrm{CD}^{+}{ }^{+} \mathrm{T}$-cell counts $>500$ cells $/ \mathrm{mm}^{3}$ were randomly assigned to begin ART immediately or defer until $\mathrm{CD}^{+}{ }^{+} \mathrm{T}$-cell counts were 350 cells $/ \mathrm{mm}^{3}$ or AIDS developed (4). All participants provided

The START (Strategic Timing of Antiretroviral Treatment) Pulmonary Substudy was supported by the NHLBI (R01 HL096453); the parent START trial was primarily supported by the National Institute of Allergy and Infectious Diseases Division of AIDS (UM1 Al068641 and UM Al120197), with additional support from the German Ministry of Education and Research, the European AIDS Treatment Network (NEAT), the Australian National Health and Medical Research Council, and the UK Medical Research Council and National Institute for Health Research. The Veterans Health Administration Office of Research and Development also provided protected research time in support of this study. The University of Minnesota served as sponsor of the study. None of the funders nor the sponsor had any input regarding the design and conduct of the study; collection, management, analysis, and interpretation of the data; preparation, review, or approval of the manuscript; or decision to submit the manuscript for publication. The views expressed in this article are those of the authors and do not reflect the views of the U.S. Government, the $\mathrm{NIH}$, the Department of Veterans Affairs, the funders, the sponsors, or any of the authors' affiliated academic institutions.

Author Contributions: K.M.K. conceived the study, obtained funding, and drafted the manuscript; K.M.K., J.V.B., and G.C. designed the study; E.B., K.M.E.F., E.N., and A.L.R. acquired the data; G.C. and J.E.C. performed the primary statistical analyses; all authors critically revised the manuscript for important intellectual content and approved the final manuscript and take responsibility for the integrity of the data and the accuracy of the data analysis.

Originally Published in Press as DOI: 10.1164/rccm.201911-2266LE on December 16, 2019 informed consent, and all site institutional review boards/ethics committees approved the substudy (ClinicalTrials.gov identifiers: NCT 01797367 and NCT 00867048).

The parent START trial demonstrated clear benefits of immediate ART on reduction of AIDS and non-AIDS events (5), at which time our primary substudy outcome of lung function decline (slope of $\mathrm{FEV}_{1}$ ) did not differ between immediate and deferred ART. When the primary outcome was analyzed in May 2015, there were two major limitations in our results. First, the initial follow-up time was relatively short, at a median of 2.0 years. Second, the duration of ART exposures was limited by only 55\% of participants in the deferred treatment group having started ART, which precluded adequately assessing difference by ART regimen on lung function decline.

After the primary results of START demonstrated the benefits of immediate treatment, ART initiation was recommended to all participants in the deferred group, and all participants in the Pulmonary Substudy continued to be followed through the end of December 2016, at which point median follow-up time was 3.9 years, thereby improving our ability to investigate the effects of differing ART regimens on lung function decline. We now present the final results of the randomized comparisons and an observational analysis comparing rate of longitudinal lung function decline among differing ART regimens.

We collected postbronchodilator spirometry before randomization and annually during follow-up. Blinded central review of all spirometry data was performed by K.M.K. Our primary outcome was rate of lung function decline, expressed as $\mathrm{FEV}_{1}$ slope in milliliters per year. We compared $\mathrm{FEV}_{1}$ slope using repeated measures mixed models (SAS 9.4), using only good-quality spirometry data and strict intention to treat. We additionally compared differing initial ART regimens to assess for potential class-specific effects. This analysis included only those who began ART before study completion and had at least one good-quality $\mathrm{FEV}_{1}$ measurement both before ART initiation and while receiving ART. All ART regimens in START contained two nucleoside/nucleotide reverse-transcriptase inhibitor (NRTI) drugs, and $90 \%$ of participants were prescribed NRTI components of tenofovir disoproxil fumarate and emtricitabine (or lamivudine), precluding meaningful comparisons between individual NRTIs. Therefore, we focused on comparisons between the nonnucleoside reversetranscriptase inhibitor (NNRTI), protease inhibitor, and integrase strand transfer inhibitor (INSTI) classes of antiretroviral drugs.

A total of 1,026 participants were randomly assigned to immediate ART $(n=518)$ or deferred ART $(n=508)$. Participants were relatively young (median age, $36 \mathrm{yr}$ ), recently diagnosed with HIV (median time since HIV diagnosis, $1.2 \mathrm{yr}$ ), with median baseline $\mathrm{CD}^{+}{ }^{+} \mathrm{T}$-cell count of 648 cells $/ \mathrm{mm}^{3}$. Of the participants, $29.1 \%$ were female, $28.3 \%$ were current smokers, and baseline lung function was generally good (median $\mathrm{FEV}_{1}$ of $96.2 \%$ of predicted), with COPD prevalence at study entry of $6.8 \%$. By the end of follow-up, ART had been initiated in $98.9 \%$ of the immediate ART group and $88.6 \%$ of the deferred group. In the immediate ART group, median $\mathrm{CD}^{+}{ }^{+}$count at the time of ART initiation was 648 cells $/ \mathrm{mm}^{3}$, and $95.5 \%$ of follow-up time was spent on ART. In the deferred ART group, median CD $4^{+}$count at the time of ART initiation was 482 cells $/ \mathrm{mm}^{3}$, and $45.1 \%$ of follow-up time was spent on ART. 